\title{
First-Principles Study of the Optical Properties of $\mathrm{SrHfO}_{3}$
}

\author{
H. Salehi, H. Tolabinejad \\ Department of Physics, Shahid Chamran University of Ahvaz, Ahvaz, Iran \\ E-mail: salehih@scu.ac.ir \\ Received March 17, 2011; revised April 15, 2011; accepted April 25, 2011
}

\begin{abstract}
The optical properties of $\mathrm{SrHfO}_{3}$ were studied by first principle using the density functional theory. The dielectric functions and optical constants are calculated using the full potential-linearized augmented plane wave (FP-LAPW) method with the generalized gradient approximation (GGA) by Wien2k package. The theoretical calculated optical properties and energy loss (EEL) spectrum yield a static refractive index of 1. 924 and a Plasmon energy of 27 eVfor cubic phase. The complex dielectric functions are calculated which are in good agreement with the available experimental results.
\end{abstract}

Keywords: Optical Properties, $\mathrm{SrHfO}_{3}$, WIEN2k, FP-LAPW, DFT, GGA

\section{Introduction}

The development of computational methods in the electronic-structure community has led to a new class of first principles approaches, based upon a full solution of the quantum mechanical ground state of the electron system within the local-density approximation(LDA)to KohnSham density functional theory (DFT) [1,2]. In principle, these methods take as their only inputs the atomic numbers of the constituent atoms. The modeling of electronic and optical properties, by means of first-principles calculations, has become a very useful tool for understanding about the structural, electronic and optical properties of the various materials.

A particularly successful application of this technique has been its use in understanding the Perovskite ferroelectric Compounds. $\mathrm{SrHfO}_{3}$ is a typical perovskite dielectric with a wide rang of technological applications. Because of its special properties related to ferroelectricity, semi conductivity. $\mathrm{SrHfO}_{3}$ is a Compound that has a Composition and lattice structure similar to $\mathrm{SrTiO}_{3}$. Although the perovskite Compound $\mathrm{SrHfO}_{3}$ has been will known for along time, theoretical studies on this compound are few [3]. Material with structure $\mathrm{ABO}_{3}$ have been the subject of extensive investigation, because of the unusual combination of their magnetic, electronic and transport properties[4,5].Their use in technological applications is also diverse, including optical wave guides, laser-host crystals, high temperature oxygen sensors, high-voltage capacitor, piezoelectric materials in actuators and so on [6,7]. Recently, stachiotti et al. [8] inves- tigated its ferroelectric instability by first-principles calculation. First-Principles calculation is one if the powerful tools for carrying out the theoretical studies of the electronic and structure properties of materials. The$\mathrm{ABO}_{3}$ Perovskite type oxides have potentials to be attractive functional materials because of their various unique Properties. Among them, alkaline. Rear-earth haf nates, $\left(\mathrm{AHfO}_{3}, \mathrm{~A}=\mathrm{Ba}, \mathrm{Sr}\right.$ or $\left.\mathrm{Ca}\right)$ have been reported $[3,9]$ as promising Candidates as scintillators used in $\gamma$-ray imaging fields, such as positron emission tomography. Besides, $\mathrm{SrHfO}_{3}$ is a promising candidate high-k dielectric oxide for the next generation of CMDS devices, due to its good physical and electrical Properties [10].

Kennedy et al. [11] concluded that $\mathrm{SrHfO}_{3}$ undergoes three phase transitions: From $300 \mathrm{k}$ to approximately 670 $\mathrm{k}$ the structure of $\mathrm{SrHfO}_{3}$ is orthorhombic with space group Pnma. By $870 \mathrm{k}$ it adopts a second orthorhombic structure with space group $\mathrm{Cmcm}$. Its then undergoes a further phase transition and is tetragonal with space group $14 / \mathrm{mcm}$ from 1000 to $1353 \mathrm{k}$. At higher temperature the structure is the ideal cubic perovskite with space group $\mathrm{Pm} \overline{3} \mathrm{~m}$. In the present work the optical properties of $\mathrm{SrHFO}_{3}$ in cubic phase have been studied using the FP-LAPW method. The results, in comparison with the previous theoretical data, are in better agreement with the experimental results.

\section{Method of Calculation}

Calculation of the optical properties, of $\mathrm{SrHfO}_{3}$ were carried out with a self-consistent scheme by solving the 
Kohn-Sham Equation using aFP-LAPW method in the framework of theDFT along with the GGA method [12, 13] by WIEN2k package [14]. In the FP-LAPW method, space is divided into two regions, a spherical "muffin-tin" around the nuclei in which radial solutions of Schrödinger Equation and their energy derivatives are used as basis functions, and an "interstitial" region between the muffin tins (MT) in which the basis set consists of plane waves. There is no pseudopotential approximation and core states are calculated self-consistently in the crystal potential. Also, core states are treated fully relativistically while valence and semi-core states are treated semirelativistically (i.e. ignoring the spin-orbit coupling). The cut-off energy, which defines the separation of the core and valance states, was chosen as $-6 \mathrm{Ry}$. The complex dielectric tensor was calculated, in this program, according to the well-known relations [15].

$$
\begin{aligned}
& \operatorname{Im} \varepsilon_{\alpha \beta}(\omega)=\frac{4 \pi \mathrm{e}^{2}}{m^{2} \omega^{2}} \\
& \sum_{c, v} \int \mathrm{d} k\left\langle c_{\boldsymbol{k}}\left|p^{\alpha}\right| v_{\boldsymbol{k}}\right\rangle\left\langle v_{\boldsymbol{k}}\left|p^{\beta}\right| c_{\boldsymbol{k}}\right\rangle \delta\left(\varepsilon_{\mathrm{c}_{\boldsymbol{k}}}-\varepsilon_{v_{\boldsymbol{k}}}-\omega\right) \\
& \operatorname{Re} \varepsilon_{\alpha \beta}(\omega)=\delta_{\alpha \beta}+\frac{2}{\pi} P \int_{0}^{\infty} \frac{\omega^{\prime} \operatorname{Im} \varepsilon_{\alpha \beta}\left(\omega^{\prime}\right)}{\omega^{\prime 2}-\omega^{2}} \mathrm{~d} \omega^{\prime}
\end{aligned}
$$

And the optical conductivity is given by:

$$
\operatorname{Re} \sigma_{\alpha \beta}(\omega)=\frac{\omega}{4 \pi} \operatorname{Im} \varepsilon_{\alpha \beta}(\omega)
$$

In Equation (1), $c_{\mathbf{k}}$ and $v_{\mathbf{k}}$ are the crystal wave functions corresponding to the conduction and the valance bands with crystal wave vector $\mathbf{k}$. In Equation (3) the conductivity tensor relating the interband current density $\mathrm{j}$ in the direction which flows upon application of an electric field $\mathrm{E}$ in direction in which the sum in Equation (1) is over all valence and conduction band states labeled by $v$ and $c$. Moreover, the complex dielectric constant of a solid is given as:

$$
\varepsilon(\omega)=\varepsilon_{1}(\omega)+i \varepsilon_{2}(\omega)
$$

Here, real and imaginary parts are related to optical constants $n(\omega)$ and $k(\omega)$ as:

$$
\begin{aligned}
& \varepsilon_{1}(\omega)=n^{2}(\omega)-k^{2}(\omega) \\
& \varepsilon_{2}(\omega)=2 n(\omega) k(\omega)
\end{aligned}
$$

The other optical parameters, such as energy-loss spectrum and oscillator strength sum rule are immediately calculated in terms of the components of the complex dielectric function [15].

\section{Results and Discussion}

The calculations first were carried out using the experimental data for lattice constants $(\mathrm{a}=7.78 \mathrm{a} . \mathrm{u})[8]$ in the cubic phase. Then by minimizing the ratio of the total energy of the crystal to its volume (volume optimizing) the theoretical lattice constants were obtained $(\mathrm{a}=7.87$ $\mathrm{au}$ ). In order to reduce the time of the calculations we used the symmetries of the crystal structure and some other approximations for simplicity. The calculation was performed with $1000 \mathrm{k}$-points inthe cubic phase.

The self-consistent process, for both phases, after 11 cycles had convergence of about 0.0001 in the eigenvalues in which for the cubic phase 388 plane waves were produced. Under these conditions the values of the other parameters were $\mathrm{G}_{\max }=14, \mathrm{R}_{\mathrm{MT}}(\mathrm{Sr})=2 \mathrm{au}, \mathrm{R}_{\mathrm{MT}}(\mathrm{Hf})=$ $2.2 \mathrm{au}, \mathrm{R}_{\mathrm{MT}}(\mathrm{O})=1.5 \mathrm{au}$. The iteration halted when the total charge adjustment was less than 0.0001 between steps.

\subsection{Dielectric Function}

In WIEN2K calculations for finding optical functions, reflactions of kramers-kronig was used. As an example in transformation kramers-kronig, without coupling spinorbit and magnetic field, the dielectric tensor cubic structure is as follows:

$$
\begin{aligned}
& \left(\begin{array}{lrc}
\operatorname{Im} \varepsilon_{x x} & 0 & 0 \\
0 & \operatorname{Im} \varepsilon_{x x} & 0 \\
0 & 0 & \operatorname{Im} \varepsilon_{x x}
\end{array}\right) \text { kramers - kronig } \\
& \left(\begin{array}{lrc}
\operatorname{Re} \varepsilon_{x x} & 0 & 0 \\
0 & \operatorname{Re} \varepsilon_{x x} & 0 \\
0 & 0 & \operatorname{Re} \varepsilon_{x x}
\end{array}\right)
\end{aligned}
$$

In this theoretical method, momentum matrix elements, for finding dielectric function and other optical properties Equation (3) is used. Because it has hexagonal closed packed structure (hcp), so due to tensor form for this structure, the selections $\varepsilon_{x x}$ (dielectric function in direction $\mathrm{x}$ ) and $\varepsilon_{z z}$ (dielectric function in direction z)are enough for calculating dielectric tensor.

We calculated optical properties of $\mathrm{SrHfO}_{3}$ in the cubic phase. The real and the imaginary parts of the dielectric functions are shown in Figure 1 for $\mathrm{SrHfO}_{3}$ in the cubic phase. In Figure, there are mainly four peaks (5.5, 7, 9.5 and $12 \mathrm{eV})$. Peaks A $(5.5 \mathrm{eV}), \mathrm{B}(7 \mathrm{eV})$ correspond mainly to the transitions from $\mathrm{O} 2 \mathrm{p}$ to $\mathrm{Hf} 5 \mathrm{~d}$ states along the $\Gamma$ direction, and peaks $\mathrm{C}(9.5 \mathrm{eV}), \mathrm{D}(12 \mathrm{eV})$ correspond mainly to the transitions from semicore bands (formed by Sr- $4 p$ and O- $2 \mathrm{~s}$ states) to conduction bands. The value of the main peak of $\varepsilon_{1}(\omega)$ curve is 6 at energy of $5.5 \mathrm{eV}$ and for $\varepsilon_{2}(\omega)$ is 6.4 at the energy equal $4 \mathrm{eV}$.

Figure 1 shows the change of $\operatorname{Re}\left(\varepsilon_{x x}\right)$ (Real part of dielectric function in the $\mathrm{x}$ direction) against photons 


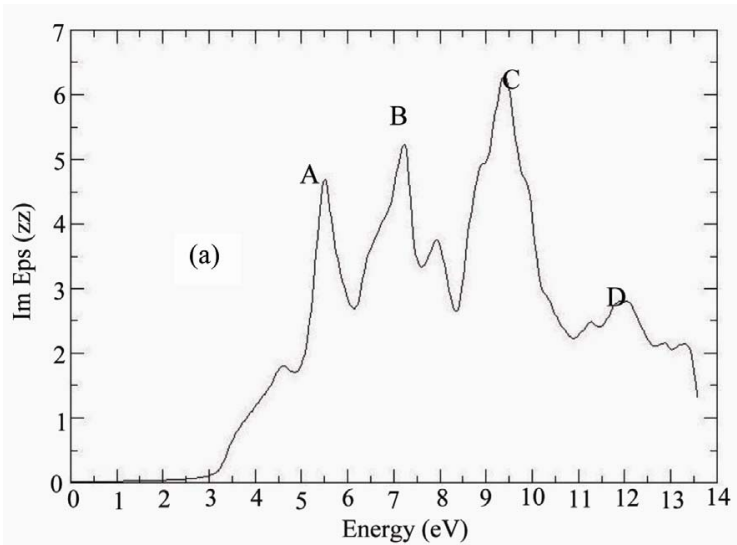

(a)

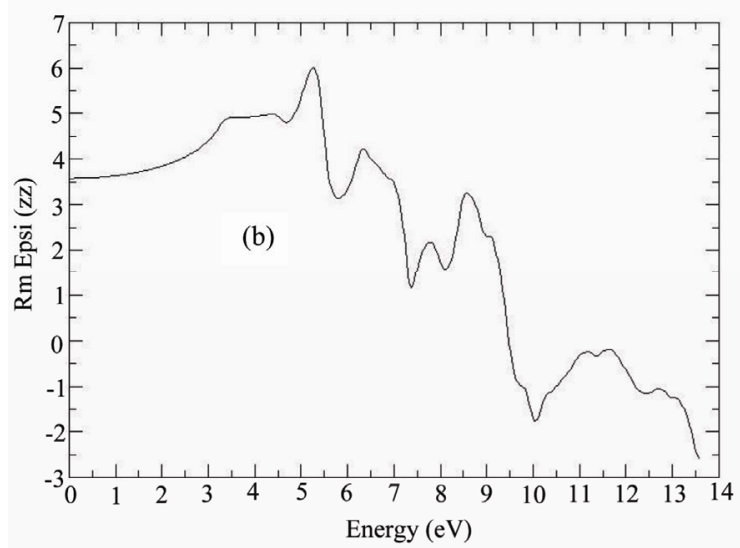

(b)

Figure 1. (a) Real and (b) imaginary part of the dielectric function for $\mathrm{SrHfO}_{3}$ in cubic phase.

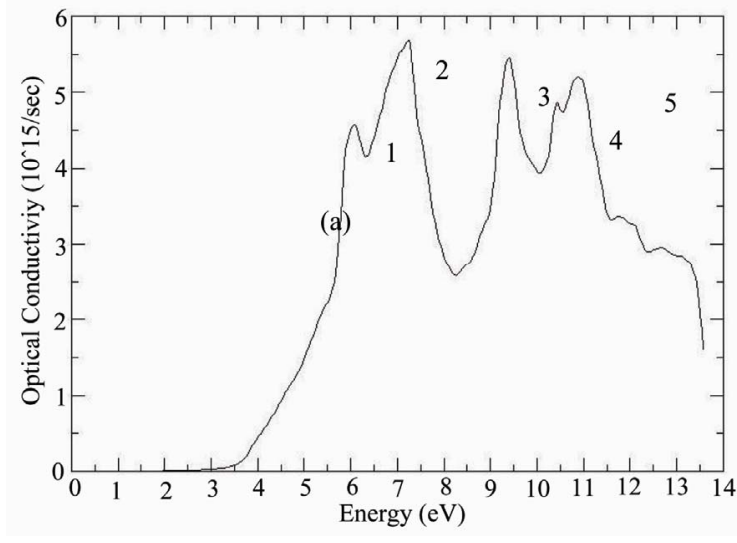

(a)

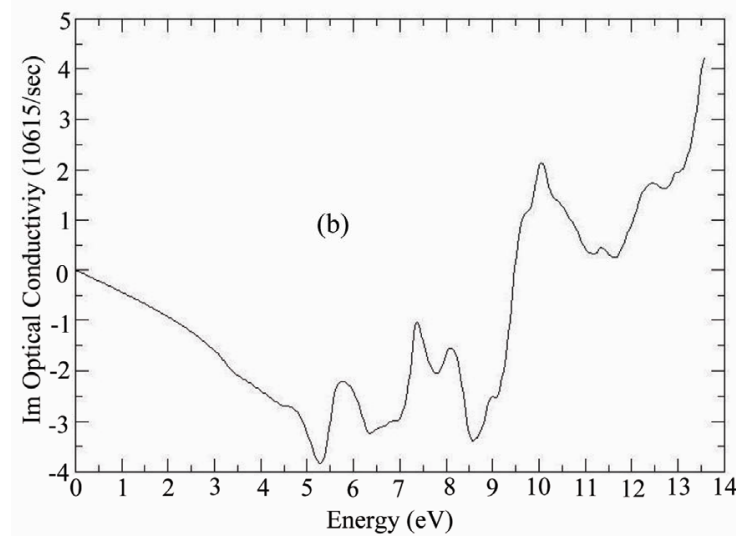

(b)

Figure 2. (a) Real and (b) imaginary part of the optical conductivity for $\mathrm{SrHfO}_{3}$ in cubic phase.

Table 1. $\mathrm{SrHfO}_{3}$ static refractive index in cubic phase calculated by various methods.

\begin{tabular}{ccccc}
\hline \multicolumn{3}{c}{ Present work } & & Other work \\
\hline & $\begin{array}{c}\text { FP-LAPWG FP-LAPWG } \\
\text { GA96 }\end{array}$ & GA91 & $\begin{array}{c}\text { FP-LAP- } \\
\text { WLDA }\end{array}$ & $\begin{array}{c}\text { Experimental } \\
{[16]}\end{array}$ \\
\hline $\begin{array}{c}\text { Refractive } \\
\text { index (n) }\end{array}$ & 1.924 & 1.9050 & 1.867 & 2 \\
$\begin{array}{c}\text { Difference with } \\
\text { exp. (\%) }\end{array}$ & 3.8 & 4.75 & 6.65 & - \\
& 3.7 & 3.63 & 3.5 & 4 \\
\hline
\end{tabular}

energy. In the zero energy level, the magnitude of dielectric function, $\varepsilon(0)$ is equal to 3.7 . By the relation $\sqrt{\varepsilon(\omega)}=n(\omega)+i k(\omega)=N(\omega)$, the root of dielectric function will give the refraction coefficient of $\mathrm{SrHFO}_{3}$ which is equal to $n=\sqrt{\varepsilon(0)}=\sqrt{3.7}=1.924 n$. The magnitude of experimental refraction coefficient for
$\mathrm{SrHFO}_{3}$ is 2 [16] which has a good agreement.

The first peak of $\operatorname{Im} \varepsilon$ is contributed to transition from the upper valence bands to the lower conduction band. Our calculated in direct $(\Gamma-R)$ band gap of $\mathrm{SrHfO}_{3}$ is about $3.7 \mathrm{eV}$.

The real part of the dielectric function $(\operatorname{Re} \varepsilon)$ follow from the Kramer-kronig relationship. All Optical constant may by derived from this.

Referring to Table 1, it can be seen that the calculated refractive index in this work is in agreement with experimental results.

\subsection{Optical Conduction}

The real and the imaginary parts of optical conductivity are shown in Figure 2 for $\mathrm{SrHfO}_{3}$ in cubic phase.

In this theoretical method, momentum matrix elements is used for finding dielectric function and other optical properties. Also we have used transformation Kramers-Kronig for calculating real part dielectric tensor. 
Notice that, occupied electron states are excited to unoccupied electron states in upper Fermi level by absorbing photons. This interband transition is called optical conduction (also called Drude transition) and photon absorption by electron is called interband absorption.

Figure 4 shows the change of real part of optical conduction (in $10^{15} \mathrm{~s}^{-1}$ ) in the $\mathrm{X}$ direction with respect to internal photon energy. As in the Figure 2, the optical conduction starts with energy of about $2 \mathrm{eV}$ and by increasing photon energy, the optical conduction will rises and in the range of 6.5 till $7 \mathrm{eV}$, will reach to the upper level. The reason of starting optical conduction, $\sigma(\omega)$, from $2 \mathrm{eV}$ energy, is the gap of energy. So the excited electrons have no enough energy to pass the energy gap, and transfer to the conduction band.

For the absorption coefficient, $\alpha(\omega)$, the absorption edge start from about $3.4 \mathrm{eV}$, corresponding to the direct $\Gamma-\Gamma$ transition. The maximum reflectivity about $13 \mathrm{eV}$, ie the minimum value $\varepsilon_{1}$ will occur. In the regime incident beam energy, has not been reached to create a new exaction mode yet or can prepar interband transition and thus waves will be completely reflectivity.

The peak function $\alpha(\omega)$ also in energy $11.5 \mathrm{eV}$ can be seen, and with this mean the most value absorption in the band gap, which the matter cannot exhibit any transparency.

In Figure 3 the optical parameters is shown for $\mathrm{SrHfO}_{3}$ in cubic phase. The static refractive index value for $\mathrm{SrHfO}_{3}$ in the cubic phase calculated in this work, and the values obtained by other methods are summarized in Table 1.

If we assume assume orientation of the crystal surface parallel to the optical axis, the reflectivity $R(\omega)$ follows directly from Fresnels formula.

$$
R(\omega)=\left|\frac{\sqrt{\varepsilon(\omega)}-1}{\sqrt{\varepsilon(\omega)}+1}\right|^{2}
$$

Expression for the absorption coefficient $I(\omega)$ now follow:

$$
I(\omega)=\sqrt{2}(\omega)\left(\sqrt{\varepsilon_{1}(\omega)^{2}+\varepsilon_{2}(\omega)^{2}}-\varepsilon_{1}(\omega)\right)^{1 / 2}
$$

\subsection{Electron Energy Loss Spectroscopy}

EELS is a valuable tool for investigating various aspects of materials. It has the advantage of covering the complete energy range including non-scattered and elastically

Tabel 2. The value transition interband.

\begin{tabular}{cccccc}
\hline Transition & First & Two & Three & Four & Five \\
\hline $\mathrm{E}(\mathrm{eV})$ & 5.5 & 6.7 & 9.5 & 10.5 & 11.2 \\
\hline
\end{tabular}

scattered electrons (Zero Loss). At intermediate energies (typically 1 to $50 \mathrm{eV}$ ) the energy losses are due primarily to a complicated mixture of single electron excitations and collective excitations (plasmons). The positions of the single electron excitation peaks are related to the joint density of states between the conduction and valence bands, whereas the energy required for the excitation of bulk plasmons depends mainly on the electron density in the solid. Here electrons, which excite the atoms electrons of the outer shell is called Valence Loss or valence interaband transitions. At higher energies, typically a few hundred $\mathrm{eV}$, edges can be seen in the spectrum, indicating the onset of excitations from the various inner atomic shells to the conduction band.In this case the fast electrons excite the inner shell electrons (Core Loss)or induce core level excitation of Near Edge Structure (ELNES) and XANES. The edges are characteristic of particular elements and their energy and height can be used for elemental analysis.

In the case of interband transitions, which consist mostly of plasmon excitations, the scattering probability for volume losses is directly connected to the energy loss function. One can then calculate the EEL spectrum from the following relations.

$$
\begin{aligned}
& \varepsilon_{\alpha \beta}(\omega)=\varepsilon_{1}+\mathrm{i} \varepsilon_{2} \text { and } \\
& \text { EELSpectrum }=\operatorname{Im}\left[-1 / \varepsilon_{\alpha \beta}(\omega)\right]=\frac{\varepsilon_{2}}{\varepsilon_{1}^{2}+\varepsilon_{2}^{2}}
\end{aligned}
$$

These peaks can, however, have different origins such as charge carrier plasmons and interband or intraband excitations. The energy of the maximum peak of Im $\left[-\varepsilon^{-1}(\mathrm{E})\right]$ at $27 \mathrm{eV}$ is assigned to the energy of the volume plasmon $\hbar \omega_{p}$. The first peak at $9 \mathrm{eV}$ and second peak at $13 \mathrm{eV}$ originates from O-2p to Hf-3d and Sr-p orbitals, respectively Figure 4. The value of $\hbar \omega_{p}$ obtained in this work and for free electron is given in Table $\mathbf{3}$.

For free electrons the plasmon energy is calculated according to the following model:

$$
\hbar \omega_{p}^{e}=\hbar \sqrt{\frac{n e^{2}}{\varepsilon_{0} m}}
$$

If we use this model, then what should be the number of valance electrons per $\mathrm{SrHfO}_{3}$ molecule, $\mathrm{N}$, used to calculate the density of valance electrons, $n$, and thus the plasmon energy in Equation (7). If we take only the contribution of $4 s^{2}, 4 p^{6}$ and $5 s^{2}$ electrons of $\mathrm{Sr}, 5 \mathrm{~s}^{2}, 5 \mathrm{p}^{6}, 5 \mathrm{~d}^{10}$ and $6 \mathrm{~s}^{2}$ of $\mathrm{Hf}$ and $2 \mathrm{~s}^{2}, 2 \mathrm{p}^{4}$ of $\mathrm{O}$ (ignoring the contribution of $5 s^{2}$ and $2 s^{2}$ electron of $\mathrm{Sr}$ and $\mathrm{O}$ atom respectively) and the free and electron plasmon energy will be $31 \mathrm{eV}$. Otherwise, with all valance electrons of $\mathrm{Sr}, \mathrm{Hf}$ and $\mathrm{O}$ atoms, the free electron plasmon would be $38.6 \mathrm{eV}$. We will also see from sum rule is a reasonable value for the valance electrons per $\mathrm{SrHfO}_{3}$ molecule. 
Table 3. $\mathrm{SrHfO}_{3}$ plasmon energy $\left(\hbar \omega_{p}\right)$ of the energy loss function in cubic phase calculated by this method and free electron.

\begin{tabular}{ccccccc}
\hline & Present work & Present work & Present work & Present work & Present work & Other work \\
\hline & GGA96 & GGA91 & LDA & Free electron (ignoringSr-5s and O-2s states) & Free electron & Theoretical [17] \\
Plasmon energy $(\mathrm{eV})$ & 27 & 27.73 & 26.2 & 31 & 38.6 & 27.3 \\
\hline
\end{tabular}

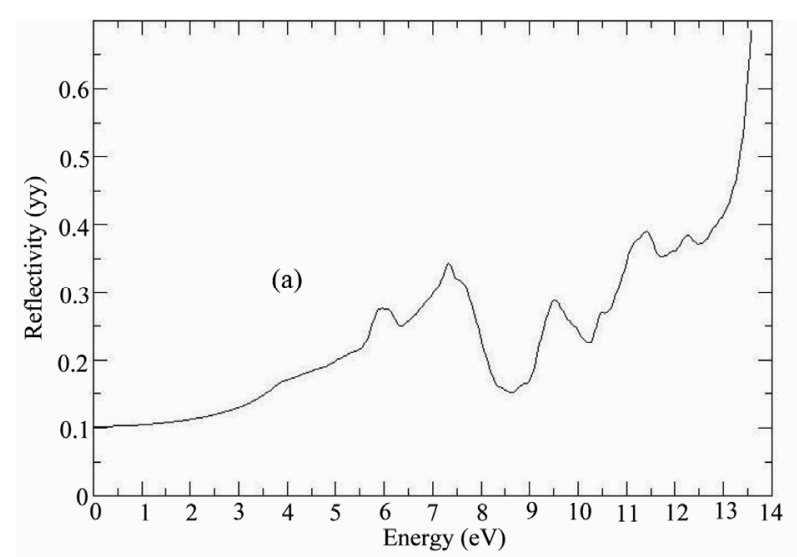

(a)

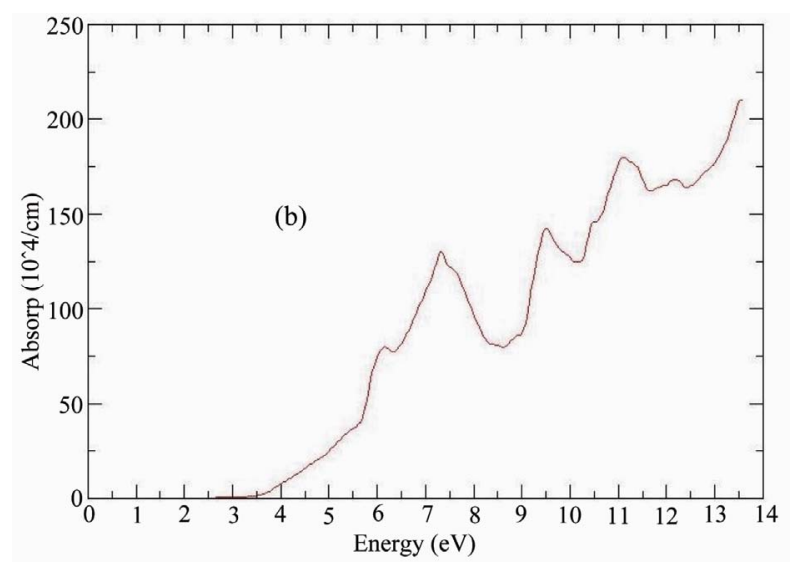

(b)

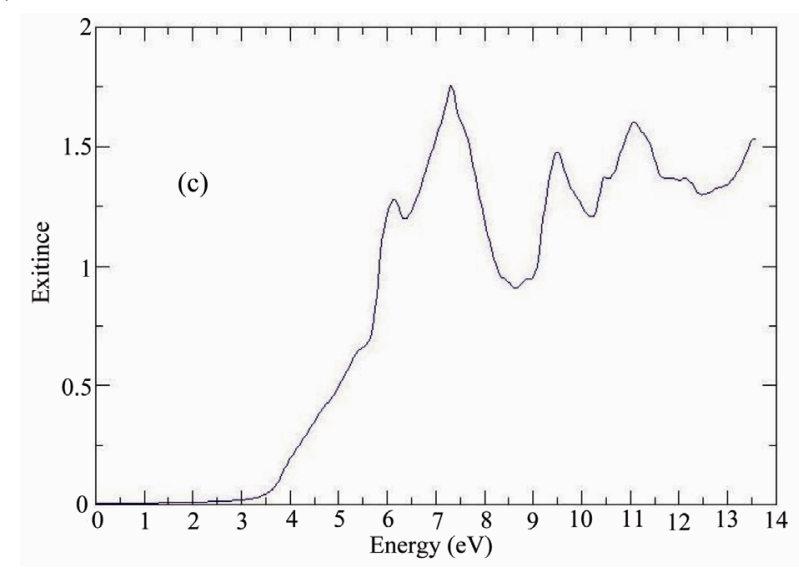

(c)

Figure 3. The calculated optical parameters of the cubic $\mathrm{SrHfO}_{3}$ as a function of the photon energy (eV). (a), absorption coefficient, $\alpha(\omega)$; (b), reflectivity coefficient $R(\omega)$ and (c), extinction coefficient $k(\omega)$.

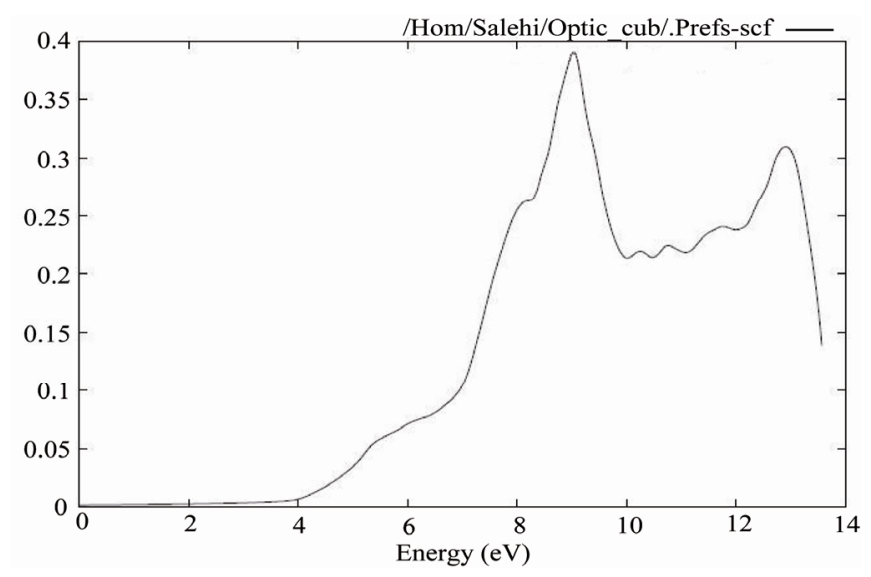

Figure 4. Electron energy Loss spectroscopy for $\mathrm{SrHfO}_{3}$ cubic phase. 


\section{Conclusions}

We have calculated the optical properties of $\mathrm{SrHfO}_{3}$ in cubic phases using the FP-LAPW method with the generalized gradient approximation (GGA). Thecalculations show a static refractive index of 1.924 and an EEL spectrum of $27 \mathrm{eV}$ for the cubic phase. Ignoring the contribution of $5 s^{2}$ and $2 s^{2}$ electron of $\mathrm{Sr}$ and $\mathrm{O}$ atom respectively, the free electron plasmon energy will be $38.6 \mathrm{eV}$. It seems that is a reasonable value for the valance electrons per molecule. The calculated results show a good agreement with the other data.

\section{References}

[1] J. P. Perdew, J. A. Chevary, et al., Phys.Rev.B, Vol. 46, No., 1992, pp. 6671-6687.

[2] J. P. Perdew, Physical. B, Vol. 172, No. , 1991, pp. 1-6.

[3] Y. M. Ji, D. Y. Jiang, Z. H. Wu, T. Feng and J. L. Shi, Mater.Res.Bull., Vol. 40, No., 2005, pp. 1521

[4] I. R. Shein, V. L. Kozhevnikov and A. L. Ivanovskii, Solid State Sci. Vol.10, No., 2008, pp. 217.

[5] C. M. I. Okoye, J. Phys.: Condens. Matter, Vol. 15, No., 2003, pp. 5945.

[6] S. Lin, Z. Xiu, J. Liu, F. Xu, W. Yu, J. Yu and G. Feng, J. Alloy. Compd, Vol. 457, No., 2008, pp. L12.

[7] V. M. Longo, L. S. Cavalcante, A. T. de Figneiredo, L. P.
Santos, E. Longo, J. A. Varela, J. R. Sambrano, C. A. Paskocimas, F. S. De Vicente and A. C. Hernandes, Appl. Phys. Lett. Vol. 90, No., 2007, pp. 091906.

[8] M. G. Stachiotti, G. Fabricius, R. Alonso and C. O. Rodriguet, Phys.Rev.B, Vol. 58, No., 1998, pp. 8145.

[9] R. Vali, Solid State Communications, Vol. 148, No. , 2008, pp. 29-31.

[10] C. Rossel, M. sousa, C. Marchiori, et al. Microelec. Eng Vol. 84, No., 2007, pp. 1869.

[11] B. J. Kennedy, C. J. Howard and B. C. Chakoumakos, Phys.Rev.B, Vol. 60, No., 1999, pp. 2972.

[12] J. P. Perdew, J. A. Chevary, S. H. Vosko, K. A. Jackson, M. R. Pederson, D. J. Singh and C. Fiolhais, Phys. Rev. B Vol. 46, No., 1992, pp. 6671-6687.

[13] M. Peterson, F. Wanger, L. Hufnagel, M. Scheffler, P. Blaha and K. Schwarz, Computer Physics Communications, 126, (2000) 294-309. doi:10.1016/S0010-4655(99)00495-6

[14] P. Blaha and K. Schwarz, WIEN2k, Vienna University of Technology Austria, 2009.

[15] F. Wooten, "Optical Properties of Solids," Academic Press, New York, 1972.

[16] M. Sousa, C. Rossel, C. Marchiori, D. Caimi, J. P. Locquet, D. J. Webb, K. Babich, J. W. Seo and C. Dieker, J. Appl. Phys. Vol. 102, No., 2007, pp. 104103.

[17] Z. Feng, H. ShouxinCui and C. HengshuaiLi, Journal of Physics and Chemistry of Solids Vol. 70 No. , 2009, pp. 412-416. 